\title{
Influence of partial or total replacement of glycerol by alternative cryoprotectants in Gent freezing extender on post-thaw sperm quality in stallions
}

*Autor correspondente

e-mail: rodrigocavalos@yahoo.com.br

\begin{abstract}
Although glycerol is the cryoprotectant most commonly used in stallions, it has also a considerable toxicity for equine sperm. It was the aim of this study to analyse the quality of frozen-thawed stallion semen after complete or partial replacement of glycerol in the freezing extender by alternative cryoprotectants. We hypothesized that partial or total replacement of glycerol by cryoprotectants occurring in cold-resistant frog, insect or plant species results in similar or better semen quality after freezing-thawing. As basic medium, the commercial Gent basic extender was used and either supplemented with glucose and urea, trehalose and proline, or trehalose and betaine. Based on a series of preliminary experiments, semen was frozen in either commercial Gent cryopreservation extender (Gent control), Gent glucose-urea extender or a Gent combined extender (glucoseurea, trehalose-betaine and trehalose-proline; volume ratio of 2:1:2) in a computer-controlled rate freezer. After freezing-thawing, semen was analysed for motility, membrane integrity, phosphatidylserine translocation, mitochondrial membrane potential and chromatin condensation. No differences between Gent control and Gent glucose-urea extender were seen, while all endpoints except DNA integrity were negatively affected in Gent combined extender (e.g., progressive motility: Gent $49.2 \pm 3.7$, Gent glucose-urea $46.5 \pm 4$.6, Gent combined 24.4 $\pm 2.8 \%$; $\mathrm{P}<.001$ ). In conclusion, glycerol concentration in a commercial freezing extender for equine spermatozoa can be successfully reduced when urea as an additive cryoprotectant is added and the glucose concentration is elevated. However, total glycerol replacement with urea, betaine, proline and trehalose was less successful.
\end{abstract}

Keywords: Cryoprotectants. Glycerol. Horse.

Acknowledgements: this research was supported by CAPES (Coordenacão de Aperfeiçoamento de Pessoal de Nível Superior) Scholarship to Rodrigo Arruda de Oliveira. 